УДК 528.002.5

МАТЕМАТИКО-СТАТИСТИЧЕСКОЕ ИССЛЕДОВАНИЕ ВЛИЯНИЯ ПОГРЕШНОСТЕЙ ИСХОДНЫХ ДИРЕКЦИОННЫХ УГЛОВ И ПОГРЕШНОСТЕЙ УГЛОВ ПОВОРОТА НА НАДЕЖНОСТЬ ПОЛИГОНОМЕТРИЧЕСКИХ ХОДОВ

Студ. К.П. Лоцман

МАТЕМАТИЧНО-СТАТИСТИЧНІ ДОСЛІДЖЕННЯ ВПЛИВУ ПОХИБОК ВИХІДНИХ ДИРЕКЦІЙНИХ КУТІВ І ПОХИБОК КУТІВ ПОВОРОТУ НА НАДІЙНІСТЬ ПОЛІГОНОМЕТРИЧНИХ ХОДІВ

Студ. К.П. Лоцман

\title{
MATHEMATICAL AND STATISTICAL STUDY INFLUENCE ERROR IN THE INITIAL DIRECTIONAL ANGLES AND ROTATION ANGLES ERRORS ON RELIABILITY POLYGONOMETRIC MOVES
}

Stud. K. Locman

В статье проведено исследование распределения погрешностей при расчетах дирекционньх углов для построения полигонометрических ходов. Предвычисление точности дирекционных углов проведено по специальной программе «Генератор случайных чисел». Установлено, что погрешности исходных дирекционных углов зависят от координат средних точек. Установлено, что изогнутые 
полигонометрические ходы имеют большее значение погрешностей по сравнению с прямыми. Определень дальнейшие направления в разработке математико-статистических моделей полигонометрических ходов.

Ключевые слова: дирекционный угол, полигонометрический ход, погрешность.

У статті проведено дослідження розподілу похибок при розрахунках дирекиійних кутів для побудови полігонометричних ходів. Передобчислювання точності дирекційних кутів проведено за спеціальною програмою "Генератор випадкових чисел». Встановлено, щу похибки вихідних дирекиійних кутів залежать від координат середніх точок. Встановлено, що вигнуті полігонометричны ходи мають більше значення похибок у порівнянні з прямими. Визначено подальші напрями у розробленні математично-статистичних моделей полігонометричних ходів.

Ключові слова: дирекційний кут, полігонометрічний хід, похибка.

The paper investigated the distribution of errors in the calculation of directional angles to build polygonometric moves. Precompute the accuracy of directional angles is given by the special program " random number generator "found that error in the initial directional angles depends on the coordinates of the midpoints. Established that curved polygonometric strokes are more significant errors compared to direct. Determining the direction of further development in the mathematical and statistical models polygonometric moves.

Keywords: azimuth, traverse, error.

Постановка проблемы. Создание геодезических сетей эффективнее выполнять на базе применения математико-статистических моделей. При этом желательно при расчете использовать прогрессивные методы построения таких сетей: полигонометрические ходы с боковыми пунктами. Это позволяет сократить число исполнителей полевых работ, существенно повысить точность угловых и линейных измерений. Для определения дирекционных углов в геодезических сетях сгущения очень эффективен метод, приведенный в статье, что показано в расчетах.

Актуальной задачей является поиск математико-статистических моделей, упрощающих расчет погрешностей угловых измерений $[1,2]$.

Анализ последних исследований и публикаций. Технология создания геодезических сетей, а также математикостатистическое исследование влияния погрешностей исходных дирекционных углов поворота на точность полигонометрических ходов детально проанализировано в работах В.Д.Большакова, Ю.И. Маркузе, В.М.Лобачева, А.В.Маслова, М.М. Машимова, В.З.Пащенкова, Ю.К. Неумывакина,

А.С. Смирнова, А.И. Спиридонова и др.

Главным требованием создания моделей расчета погрешностей является их точность.

На основе теоретических исследований влияния погрешностей исходных дирекционных углов на точность полигонометрических ходов сделан вывод о том, что при одинаковой точности исходных дирекционных углов и углов поворота в ходах влияние первых на дирекционные углы сторон во всех случаях меньше влияния погрешностей углов поворота. Можно с уверенностью сказать, что определить исходный угол при проложении полигонометрических ходов не составляет труда.

С этой целью в процессе построения геодезических сетей сгущения выполнены экспериментальные определения дирекционных углов. Основной причиной появления погрешностей угловых измерений являются недостаточно качественные математические модели обработки угловых измерений.

Постановка задачи. Целью статьи является исследование существующих способов расчета дирекционных углов и погрешностей, а также поиск новых подходов к решению задачи: аналитический расчет координат полигонометрического хода.

Основной материал исследования. Под математико-статистическим исследованием имеется в виду, во-первых, подтверждение правильности полученных формул для вытянутых ходов, а во-вторых, исследование влияния погрешностей исходных дирекционных углов и углов поворота на точность изогнутых полигонометрических ходов. 
Исследование выполнено для одного прямолинейного хода, расположенного по оси абсцисс, и двух изогнутых ходов. Во всех трех случаях длины ходов $L=12$ км, число сторон $n=8$, длины сторон $\mathrm{S}=1,5$ км [1].

Ряды случайных чисел получали по специальной программе «Генератор случайных чисел».

В качестве заданной средней квадратической погрешности (стандарта) дирекционных углов исходных сторон и углов поворота принята $m_{\alpha}=m_{\beta}=5 "$, а в качестве предельной

$$
\Delta_{\alpha}=\Delta_{\beta}=2,5 m_{\alpha}=2,5 m_{\beta}=2,5^{\prime \prime} m_{\beta}=12,5^{\prime \prime} .
$$

Эталонными служили значения дирекционных углов и координат пунктов, полученные в результате строгого уравнивания ходов без изменения исходных данных, углов поворота и длин сторон, значения которых приняты за исходные [2].

Изменяя сначала исходные дирекционные углы, а затем углы поворота ходов как случайные величины, подчиняющиеся нормальному распределению, каждый из полигонометрических ходов строго уравнивали, получая новые значения дирекционных углов, сторон и координат пунктов.

Для каждого заданного значения средней квадратической погрешности получено 60 случайных чисел в пределах от нуля до предельного значения этой погрешности, в соответствии с которыми изменялись исходные дирекционные углы и углы поворота. Таким образом, каждый из трех полигонометрических ходов уравнивали 120 раз: 60 вариантов с изменением исходных дирекционных углов и 60 вариантов с изменением углов поворота.

Оценка влияния погрешностей исходных дирекционных углов на элементы изогнутого полигонометрического хода 1 при заданных средних квадратических $m_{\alpha}=5$ " и предельных погрешностях $\Delta_{\alpha}=2,5 m_{\alpha}=12,5$ " приведены в табл. 1,2 .

В табл. 1 приведены случайные погрешности исходных дирекционных углов $\Delta_{\alpha_{\text {зад }}}$, по которым вычислены эмпирические средние квадратические погрешности $m_{\alpha_{\text {эмm }}}$ дирекционных углов исходных сторон $B F$ и $E A$ для суждения о соответствии вырабатываемых случайных чисел нормальному распределению.

В табл. 3 приведены выданные погрешности абсцисс $\Delta_{\mathrm{x}}$ и ординат $\Delta_{\mathrm{y}}$, по которым для каждой точки хода вычислены средние квадратические погрешности абсцисс $m_{x}$, ординат $m_{y}$ и положения исходных точек.

Также приведены погрешности $\Delta_{\alpha}$, по которым вычислены средние квадратические погрешности дирекционных углов $m_{\alpha}$ каждой из сторон хода.

Во всех случаях расхождения между $m_{\alpha}$ и $m_{\alpha_{\text {ми }}}$ не превышали величины $m_{m_{\alpha}}=5 / \sqrt{2 \cdot 60}=0,5^{\prime \prime}$. Это свидетельствует о близости заданных и полученных эмпирически средних квадратических погрешностей исходных дирекционных углов и углов поворота.

Таблица 1

\begin{tabular}{|c|c|c|c|c|c|c|c|c|c|}
\hline \multirow{2}{*}{ 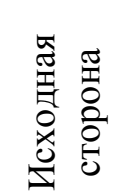 } & \multicolumn{9}{|c|}{ Заданная случайная погрешность исходного дирекционного угла } \\
\hline & 1 & 2 & 3 & 4 & 5 & 6 & 7 & 8 & 9 \\
\hline$B-F$ & $-4,3$ & $-5,1$ & $+7,9$ & $-2,4$ & $+3,0$ & $-4,8$ & $-8,8$ & $+0,3$ & $+1,3$ \\
\hline$E-A$ & $+6,1$ & $+5,6$ & $-2,7$ & $-3,2$ & $-1,3$ & $-1,8$ & $+0,8$ & $-9,0$ & $-1,1$ \\
\hline
\end{tabular}


Таблица 2

\begin{tabular}{|c|c|c|c|c|c|c|c|c|}
\hline \multirow{2}{*}{ 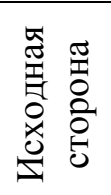 } & \multicolumn{6}{|c|}{$\begin{array}{c}\text { Заданная случайная погрешность исходного } \\
\text { дирекционного угла }\end{array}$} & \multirow{2}{*}{$\sum \Delta_{\alpha_{3 a \partial}}^{2}$} & \multirow{2}{*}{$m_{\alpha_{2 u n}}=\sqrt{\frac{\left[\Delta_{\alpha_{3 a \partial}}^{2}\right]}{n}}$} \\
\hline & 10 & 11 & $\ldots$ & $\ldots$ & 59 & 60 & & \\
\hline$B-F$ & $+4,7$ & $-6,7$ & $\ldots$ & $\ldots$ & $-4,8$ & $+1,8$ & 1460 & 4,9" \\
\hline$E-A$ & $-3,6$ & $+9,4$ & $\ldots$ & $\ldots$ & $+0,6$ & $+5,2$ & 1540 & 5,1 \\
\hline
\end{tabular}

Таблица 3

\begin{tabular}{|c|c|c|c|c|c|c|c|c|c|c|c|c|c|c|}
\hline \multirow{3}{*}{$\begin{array}{c}\text { Номер } \\
\text { испыта- } \\
\text { ния }\end{array}$} & \multicolumn{14}{|c|}{ Погрешности абсцисс и ординат точек, см } \\
\hline & \multicolumn{2}{|c|}{1} & \multicolumn{2}{|c|}{2} & \multicolumn{2}{|c|}{3} & \multicolumn{2}{|c|}{4} & \multicolumn{2}{|c|}{5} & \multicolumn{2}{|c|}{6} & \multicolumn{2}{|c|}{7} \\
\hline & $\Delta_{\mathrm{x}}$ & $\Delta_{\mathrm{y}}$ & $\Delta_{\mathrm{x}}$ & $\Delta_{\mathrm{y}}$ & $\Delta_{\mathrm{x}}$ & $\Delta_{\mathrm{y}}$ & $\Delta_{\mathrm{x}}$ & $\Delta_{\mathrm{y}}$ & $\Delta_{\mathrm{x}}$ & $\Delta_{\mathrm{y}}$ & $\Delta_{\mathrm{x}}$ & $\Delta_{\mathrm{y}}$ & $\Delta_{\mathrm{x}}$ & $\Delta_{\mathrm{y}}$ \\
\hline 1 & 1,3 & 2,3 & 1,6 & 3,6 & 1,4 & 4,2 & 0,8 & 4,7 & 0,1 & 4,0 & 0,5 & 3,2 & 0,6 & 2,0 \\
\hline 2 & 1,1 & 2,2 & 1,2 & 3,6 & 0,9 & 4,3 & 0,2 & 4,8 & 0,5 & 4,2 & 0,9 & 3,5 & 0,9 & 2,0 \\
\hline 3 & 0,1 & 0,3 & 0,0 & 0,5 & 0,0 & 0,5 & 0,1 & 0,6 & 0,2 & 0,5 & 0,2 & 0,5 & 0,2 & 0,3 \\
\hline 4 & 1,3 & 1,8 & 1,8 & 2,7 & 0,2 & 3,0 & 1,5 & 3,1 & 0,9 & 2,5 & 0,4 & 2,0 & 0,0 & 1,1 \\
\hline$\sum \Delta^{2}$ & 137 & 160 & 288 & 412 & 312 & 494 & 343 & 727 & 269 & 603 & 203 & 447 & 94 & 275 \\
\hline$m_{x} ; m_{y}$ & 1,5 & 1,6 & 2,2 & 2,6 & 2,3 & 2,9 & 2,4 & 3,5 & 2,1 & 3,2 & 1,8 & 2,7 & 1,2 & 2,1 \\
\hline$M$ & \multicolumn{2}{|c|}{2,2} & \multicolumn{2}{|c|}{3,4} & \multicolumn{2}{|c|}{3,7} & \multicolumn{2}{|c|}{4,2} & \multicolumn{2}{|c|}{3,8} & \multicolumn{2}{|c|}{3,2} & \multicolumn{2}{|c|}{2,4} \\
\hline
\end{tabular}

\section{Выводы:}

1) погрешности исходных дирекционных углов в наибольшей степени влияют на дирекционные углы крайних сторон, а в наименьшей степени - на дирекционные углы средних сторон, как в прямолинейных, так и в изогнутых ходах. Это подтверждают результаты теоретического исследования для прямолинейных ходов;

2) погрешности исходных дирекционных углов в наибольшей степени влияют на уравненные координаты средних точек, а в наименьший степени - на уравненные координаты крайних точек ходов, как в прямолинейных, так и в изогнутых ходах;

3) при одинаковой точности исходных дирекционных углов и углов поворота влияние погрешностей исходных дирекционных углов на дирекционные углы сторон полигонометрических ходов в общем меньше влияния погрешностей углов поворота, как в прямолинейных, так и в изогнутых ходах.

Это влияние в особенности четко прослеживается для средних сторон ходов. На соответствующих графиках видно, что влияние погрешностей исходных дирекционных углов в 3-5 раз меньше влияния погрешностей углов поворота. Это подтверждают результаты теоретического исследования о том, что при $m_{\alpha}=m_{\beta}$ влиянием погрешностей исходных дирекционных углов на дирекционные углы средних сторон можно пренебречь;

4) в прямолинейных ходах погрешности углов поворота в наибольшей степени влияют на дирекционные углы сторон, отстоящих от концов примерно на одну третью - одну четвертую часть длин ходов, а в наименьшей степени - на дирекционные углы сторон, расположенных в средней части ходов, что подтверждает выводы, сделанные на основе теоретических исследований. Влияние погрешностей углов поворота на дирекционные углы сторон в изогнутых ходах носит неопределенный характер; 5) как в прямолинейных, так и в изогнутых ходах погрешности углов поворота в наибольшей степени влияют на положение точек, расположенных в серединах ходов, а в наименьшей - на положение крайних точек ходов. Исключение составляет ход, состоящий из двух прямолинейных ветвей, расположенных под углом 90' друг к другу. В этом ходе указанное влияние, наоборот, практически равно нулю в точке излома, являющейся серединой хода. 


\section{Список использованных источников}

1. Лоцман, П.І. Геодезичний контроль геометрії залізничної колії [Текст] / П.І. Лоцман, О.С. Саяпін, Ю.В. Щербина // Інженерна геодезія: зб. наук. праць. - К., 2006. - Вип. 52. - С. 172-176.

2. Лоцман, П.И. Опыт применения траверсной полигонометрии на железнодорожной магистрали [Текст] / П.И. Лоцман, В.Ф. Сушков, А.А. Матвиенко, В.Г. Мануйленко // Вісник Дніпропетровського національного університету залізничного транспорту ім. акад. В. Лазаряна. Дніпропетровськ. 2006. - Вип. 10. - С. 45-48.

3. Лоцман, П.І. Розробка математичної моделі розподілу ваг напрямків у реперних мережах залізниць [Текст] / П.І. Лоцман, М.О. Пятигорец // Зб. наук. праць Укр. держ. акад. залізн. трансп. Харків: УкрДАЗТ, 2013. - Вип. 135. - С. 121-131.

4. Маслов, А.В. Геодезия [Текст] / А.В. Маслов, А.В. Гордеев, Б.Г. Батраков. - М.: Недра, 1980. $-616 \mathrm{c}$.

5. Машимов, М.М. Уравнение геодезических сетей [Текст] / М.М. Машимов. - М.: Недра, 1979. $-368 \mathrm{c}$.

6. Неумывакин, Ю.К. Практикум по геодезии [Текст] / Ю.К. Неумывакин, А.С. Смирнов. - М.: Недра, 1985.

Рецензент д-р техн. наук, профессор Э.С. Геворкян

Лоцман Клеоніка Павлівна, студентка п'ятого курсу будівельного факультету Української державної академії залізничного транспорту. Тел.: (057)730-10-67. E-mail: pavellcman @ rambler.ru.

K.P. Locman. fifth-year student of the Faculty of Construction Ukrainian State Academy of Railway Transport. Tel.: (057) 730-10-67. E-mail: pavellcman@ rambler.ru. 